\title{
To Explore the Association Between Coagulation Function and Cerebral Microbleeds in Patients With Intracerebral Hemorrhage
}

\section{Zhenhua Wang ( $\nabla 7310537 @ q q . c o m$ )}

Nanchang University Second Affiliated Hospital https://orcid.org/0000-0002-1316-6427

\section{Xinlan Xiao}

Nanchang University Second Affiliated Hospital

\section{Research Article}

Keywords: cerebral microbleeds, coagulant function, international normalized ratio.

Posted Date: April 19th, 2021

DOl: https://doi.org/10.21203/rs.3.rs-266084/v1

License: (c) (1) This work is licensed under a Creative Commons Attribution 4.0 International License.

Read Full License 


\section{Abstract}

Object:To verify the association between coagulation function and cerebral microbleeds(CMBs) in patients with intracerebral hemorrhage $(\mathrm{ICH})$.

Methods: A total of 193 patients underwent 3.0T magnetic resonance image(MRI) and were found $\mathrm{ICH}$,they were divided into CMBs and non-CMBs groups. Indicators of coagulation function and some other flood and clinical data like prothrombin time (PT), activated partial thromboplastin time(APTT), international normalized ratio (INR) were enrolled.univariate and multivariate analysis were used to compare the difference between the two groups and screen risk factors. One or more receiver operating characteristic(ROC) curve were used to present the predictive value of the indicators for CMBs.

Result: After a univariate analysis, the result showed that INR levels was significantly higher in the CMBs group than the non-CMBs group $[1.06(0.96,1.12)$ vs. $0.97(0.93,1.03) ; P=0.035)$, while there was no significant difference between PT, APTT, TT and FBI. To compare the baseline characteristic of the two groups showed that the age ,the proportion of a history of long-term antithrombotic treatment(AT), history of ischemic stroke(IS) and combination with a brain atrophy(BA) cases in the CMBs group was significantly higher than the non-CMBs group(each $P<0.05$ ). Multivariate logistic regression analysis showed that age and IS were independent risk factors for CMBs in patients with ICH (OR:0.967, 95\% Cl: 0.936-0.998, $P=0.036$; OR:2.016, 95\% Cl: 1.090-3.991, $P=0.044$; respectively). ROC curves indicated that the area under curve(AUC) of age and IS for CMBs in patients with $\mathrm{ICH}$ was $0.610(95 \% \mathrm{Cl}: 51.76 \%-70.32 \%)$ and $0.619(95 \% \mathrm{Cl}: 53.32 \%-68.87 \%)$, respectively.

Conclusion:Age and IS were the independent risk factor for $\mathrm{CMBs}$ in patients with $\mathrm{ICH}$, among the coagulant indicators, INR showed a significantly higher level in the CMBs group than the non-CMBs group.

\section{Introduction}

CMBs are dark-signal lesions with round or oval shape and a diameter of $2-10 \mathrm{~mm}$, that are easily discovered by susceptibility-weighted imaging (SWI) or Enhanced susceptibility-weighted angiography(ESWAN) [1]. Histological investigation showed that CMBs are tiny foci containing hemosiderin-laden macrophages and abnormal microvessels with blood leakage[2].The prevalence of CMBs in patients with spontaneous intracerebral hemorrhage $(\mathrm{ICH})$ is as high as $60 \%[3]$, and both the occurrence and recurrence of $\mathrm{ICH}$ are positively correlated with $\mathrm{CMBs}[4-5]$,thus, exploring the causes of CMBs may be conducive to reduce the occurrence of $\mathrm{ICH}$.

In recent years, some researchers reported that patients have a history of long-term anticoagulants treatment may lead to a raise of CMBs[6-8],however so far, the researches about the relationship between coagulant function and CMBs is still insufficient,there is still no consensus in the casual relationship between abnormal coagulation function and CMBs,thus, based on the previous studies, we 
conducted a study aimed to validate the direct relationship between coagulation function and CMBs in patients with $\mathrm{ICH}$,and provide a reference to physicians.

\section{Method And Material}

A total of 221 patients were enrolled in the study,finally 193 cases were eligible,and all the patients or their relatives signed the inform consent.Inclusion criteria were as follows:(1) all patients were diagnosed as acute $\mathrm{ICH}$ through $\mathrm{CT}$ or MRI,(2) Each bleeding volume of $<30 \mathrm{ml}$ according to the simple formula $A B C / 2$ [9],which was calculated in the CT or MRI imaging.(3) magnetic resonance imaging (MRI),including SWI sequence or ESWAN sequence, were conducted within 7 days after admission; (4) a study-related blood test had been completed within 24 hours after hospital admission.

Exclusion criteria were as follows: (a) cases age $<18$ years ; (b) cerebral hemorrhages caused by intracerebral tumors, trauma, severe infection and vascular malformations; (c) patients combined with a history of hematological disease; (e) patients were after a craniotomy.

\subsection{Observation parameters}

The following information was collected from the eligible patients:age, gender, weight,Body Mass Index(BMI), whether combination with a brain atrophy(BA), history of correlative disease (hypertension and diabetes), history of long-term an antithrombotic treatment(anticoagulant,antiplatelet or thrombolytic medicament)(AT),history of ischemic stroke(IS), systolic blood pressure, diastolic blood pressure.Information from blood preparation included:platelet (PLT), creatinine(CR), urea nitrogen(BUN), fasting blood glucose(GLU), glycation hemoglobin(GHB), blood uric acid(UA), alanine aminotransferase(ALT), aspartate aminotransferase(AST) and homocysteine(HCY).Coagulation function indicators included: the levels of fibrinogen (FBI), prothrombin time $(\mathrm{PT})$, activated partial thromboplastin time(APTT), thrombin time(TT) and international normalized ratio (INR).

\subsection{Judgement criteria and scanning conditions of CMBs}

All the patients underwent multi-sequence imaging protocol on a 3.0T MRI system(Discovery 750; GE Healthcare, Milwaukee, WI, USA), with an 8-channel head coil(GE Healthcare, Chicago, IL, USA). TIweight,T2-weight,diffusion-weighted MRI, and a SWI or ESWAN were collected for analysis. SWI sequence, our scanning conditions as the following: repetition time of $26 \mathrm{~ms}$, echo time of $20 \mathrm{~ms}$, a field of view(FOV):240 × $192 \mathrm{~mm}$, slice thickness, $2 \mathrm{~mm}$; a flip angle of $15^{\circ}$, and a matrix size, $256 \times 256$; a Voxel of $0.5 \times 0.5 \times 1.2 \mathrm{~mm}$, and a scanning time about $11 \mathrm{~min}$.

ESWAN, we applied the following conditions:echo time of the first echo (TE1), $5 \mathrm{~ms}$; echo separation, 5.1$5.2 \mathrm{~ms}$; repetition time (TR), $75 \mathrm{~ms}$; flip angle, $30^{\circ}$; receiver bandwidth of $62.5 \mathrm{kHz}$; slice thickness, $2 \mathrm{~mm}$; matrix, $416 \times 356$; FOV:220 $\times 220$ mm; the number of excitations (NEX), 0.7; flow compensation; parallel imaging array spatial sensitivity encoding technique (ASSET) with an acceleration factor of 2; number of slices, 64; and scan time, 7:50 (min:s). 


\subsection{Statistic methods}

For comparing the difference of CMBs group and non-CMBs group,SPSS 22.0 (IBM, Armonk, NY, USA) was used for data analysis. Quantitative data were presented as means \pm standard deviation (SD) or as medians with interquartile range (IQR) when the data showed a non-normal distribution and the counting data were expressed by percentage.The Kolmogorov-Smirnov normality test was used to examine the normality of the data distribution.According to the nature of the data,Chi-squared,t-tests or MannWhitney U-test was utilized to analyse.The reported statistical significance levels were all two-sided, with statistical significance set at 0.05.A multivariate logistic regression analysis was used for correlation analysis between CMBs and related variables with a significance level of 0.05 , a ROC curve was used to evaluate independent predictive values of the related variables on CMBs.

\section{Results}

\subsection{Comparison of baseline characteristics}

We were enrolled 193 patients which meet the inclusion and exclusion criteria from our institution between 2017 to 2020, among these, refer to MRI imaging,there were 117 patients with CMBs (61.9\%).According to CMBs occurrence or absence,patients were divided into CMBs and non-CMBs groups. The proportion of patients with AT,IS and BA in the CMBs group were significantly higher than that in the non-CMBs group $(28.0 \%$ vs $13.7 \%, P=0.014 ; 60.8 \%$ vs $38.2 \%, \mathrm{P}=0.009 ; 25.6 \%$ vs $10.9 \%, P=$ 0.043 ,respectively), and there was no significant difference between the two groups in other baseline characteristics( each $P>0.05$, see Table 1).

\subsection{Comparison of coagulation function and other blood parameters}

The comparison results showed that compared with non-CMBs group,CMBs group had significant longer INR (1.47 \pm 2.81 vs. $1.04 \pm 0.24 ; P=0.0038)$, and compared the PLT,PT, APTT, and FBI in the CMBs group and non-CMBs group; there were non significant difference $(P>0.05)$. Comparing the PLT, CR, BUN, GLU, GHB, UA, ALT, AST and HCY were presented without a significant difference between the two groups (each $P>0.05$; as showed in Table 2).

\subsection{Independent risk factors and its predictive value of CMBs}

INR,IS,age,AT and BA, which were showed significant difference between the two groups, were analyzed by multivariate logistic regression,the results showed that only age and IS have a significant effect on predicting the occurrence of $\mathrm{CMBs}$ (each $P<0.05$ ). Using age and IS as variate respectively, the occurrence 
of CMBs as dependent variate,the ROC analysis curve was plotted(as showed in Fig. 1). The results demonstrated that the older patients and patients with IS have more possible occurrence of CBMs,the AUC of age is $0.610(95 \% \mathrm{Cl}: 51.76 \%-70.32 \%)$, with the sensitivity of $81.82 \%$ and the specificity of $36.36 \%$. The AUC of IS is $0.619(95 \% \mathrm{Cl}: 53.32 \%-68.87 \%)$, with the sensitivity of $60.83 \%$ and the specificity of $61.82 \%$.

\section{Discussion}

Through a comparison between CMBs and non-CMBs groups,we found that age and IS were significantly associated with $\mathrm{CMBs}$, and the older patients is more possibly occur $\mathrm{CMBs}$, this result was consistent with some previous studies: The research of Poels MMF et al showed that there is significant associations between CMBs and advanced age, as well as hypertension[10].Rotterdam Scan Study described CMBs in 1,062 older subjects,CMBs were detected in $17.8 \%$ of patients aged $60-69$, in $31.3 \%$ of patients aged $70-$ 79 , and in $38.3 \%$ of patients aged $80-97[11]$. The prevalence of multiple CMBs was also found to increase significantly with age[12].A study from van Elderen et al.[13]demonstrated the brain atrophy is age-associated,so in our study,brain atrophy has been enrolled to analysis,the comparison between the two groups showed that a significant difference but it's not a independent predictor of CMBs.

Previous research about the prevalence of CMBs in IS patients were variety[14-16].The research of Kim et al.[17] showed the prevalence of CMBs in elderly subjects with no history of cerebrovascular disease is around $5 \%$, but is much higher in patients with ischemic or hemorrhagic stroke.In our study,the result showed that patients with a history of IS is an independent risk factor for CMBs,that is consistent with their finding.

The study of Lee et al. [18]reported that patients with ICH after warfarin treatment yielded a conclusion that increase in INR aggravated the occurrence of CMBs. The study of Zhang, $\mathrm{H}$ et al.[19]with 174 cases found that APTT was an independent risk factor for CMBs in patients with intracerebral hemorrhage,but another study conducted by Liu et al.[20]showed that the levels of APTT and TT were without a significant difference between CMBs group and non-CMBs group. In our study, 193 cases was enrolled and the result showed INR was the only coagulation function index which has a significant difference between CMBs group and non-CMBs group,but in the subsequent logistic regression analysis showed that it was not a independent predictor of CMBs,these previous studies indicates that there may be a direct relationship between $\mathrm{CMBs}$ and coagulation function,but consensus was still insufficient in confirming which kind of coagulation index is or are the optimal representation.

Many previous researches reported that patients on antithrombotic treatment with a higher risk of hemorrhagic complications,similarly, CMBs were found to be more frequent and extensive in patients with aspirin-associated $\mathrm{ICH}[21]$,in a cross-sectional study, CMBs were more common in patients underling an antithrombotic treatment, and aspirin use was found to be related to a lobar location[22].reference to these previous studies, a long-term history of antithrombotic treatment were included in our study and there was a significant difference between the two groups though its prediction effect was not ideal,and 
Spearman correlation analysis was used between INR and AT,the result showed the correlation coefficient is $0.235, P<0.05$,indicated that the direct relationship between the two factors.

Limitation is inevitable in this study:First,our sample size is relatively small,and only Asian patients were included in the study,non-Asian patients may yield a different association between CMBs and coagulation function, a multicentre research is expected conducting in the future.Second,our study proved that antithrombotic treatment has an association with the occurrence of CMBs,but we haven't discriminated which kind of it(anticoagulant,antiplatelet or thrombolytic drugs), it deserve to do a more explicit analysis. Third,our study mainly inflected the phenomenon that some coagulant factor affected CMBs of patients with ICH,however, so far there is still lack of some mechanism studies to illustrate the phenomenon,a targeted study will be needed in the future.

\section{Conclusion}

Our study showed that age and a history of ischemic stroke are independent risk factors for CMBs respectively.The study also showed that INR levels was significant higher in CMBs group than non-CMBs group,together with previous studies,we can demonstrate that there may be a direct relationship between CMBs and coagulant function in patients with $\mathrm{ICH}$.

\section{Declarations}

\section{Ethics approval and consent to participate}

Ethical approval was obtained from the institutional Human Research Ethics Committee. The need for informed consent was waived due to the retrospective nature of the study.

\section{Consent for publication}

Yes

\section{Availability of data and materials}

The data will be available via connecting the corresponding author.

\section{Competing interests}

The authors declare that they have no conflict of interest.

\section{Funding}

No apply

\section{Acknowledgements}




\section{References}

1. Renard, D., Tatu, L., \& Thouvenot, E. (2018). Infratentorial Cerebral Microbleeds in Patients with Cerebral Amyloid Angiopathy. Journal of stroke and cerebrovascular diseases: the official journal of National Stroke Association, 27(9), 2534-2537. https://doi.org/10.1016/j.jstrokecerebrovasdis.2018.05.015.

2. Rosenblum, W. I. (1999). Small chronic hemorrhages and ischemic lesions in association with spontaneous intracerebral hematomas. Stroke, 30(12), 2761-2762. https://doi.org/10.1161/01.str.30.12.2759-b.

3. Wilson, D., Charidimou, A., Ambler, G., Fox, Z. V., Gregoire, S., Rayson, P., \& Werring, D. J. (2016). Recurrent stroke risk and cerebral microbleed burden in ischemic stroke and TIA: A meta-analysis. Neurology, 87, 1501-1510. https://doi.org/10.1212/WNL.0000000000003183.

4. Charidimou, A., Imaizumi, T., Moulin, S., Biffi, A., Samarasekera, N., Yakushiji, Y., ... Werring, D. J. (2017). Brain hemorrhage recurrence,small vessel disease type, and cerebral microbleeds: A metaanalysis. Neurology, 89, 820-829. https://doi.org/10.1212/WNL.0000000000004259.

5. Lee, S. H., Kim, B. J., \& Roh, J. K. (2006). Silent microbleeds are associated with volume of primary intracerebral hemorrhage. Neurology, 66, 430-432. https://doi.org/10.1212/01.wnl.0000196471.04165.2b.

6. Liang, Y., Song, Q., Jiao, Y., Lin, J., Qu, H., Zhao, S., ... Zhao, M. (2018).Cerebral microbleeds and the safety of anticoagulation in ischemic stroke patients: A systematic review and meta-analysis.Clinical Neuropharmacology, 41, 202-209. https://doi.org/10.1097/WNF.0000000000000306.

7. Orken, D. N., Uysal, E., Timer, E., Kuloglu-Pazarcı, N., Mumcu, S., \& Forta, H. (2013) New cerebral microbleeds in ischemic stroke patients on warfarin treatment: Two-year follow-up. Clinical Neurology and Neurosurgery, 115, 1682-1685. https://doi.org/10.1016/j.cline uro.2013.03.004.

8. Saito, T., Kawamura, Y., Sato, N., Kano, K., Takahashi, K., Asanome, A., \& Hasebe, N. (2015). Nonvitamin $\mathrm{K}$ antagonist oral anticoagulants do not increase cerebral microbleeds. Journal of Stroke and Cerebrovascular Diseases, 24, 1373-1377.

https://doi.org/10.1016/j.jstrokecerebrovasdis.2015.02.018.

9. Kothari, R. U., Brott, T., Broderick, J. P., Barsan, W. G., Sauerbeck, L. R., Zuccarello, M., \& Khoury, J. (1996). The ABCs of measuring intracerebral hemorrhage volumes. Stroke, 27(8), 1304-1305. https://doi.org/10.1161/01.str.27.8.1304.

10. Poels, M. M., Vernooij, M. W., Ikram, M. A., Hofman, A., Krestin, G. P., van der Lugt, A., \& Breteler, M. M. (2010). Prevalence and risk factors of cerebral microbleeds: an update of the Rotterdam scan study. Stroke, 41(10 Suppl), S103-S106. https://doi.org/10.1161/STROKEAHA.110.595181.

11. Vernooij, M. W., van der Lugt, A., Ikram, M. A., Wielopolski, P. A., Niessen, W. J., Hofman, A., Krestin, G. P., \& Breteler, M. M. (2008). Prevalence and risk factors of cerebral microbleeds: the Rotterdam Scan 
Study. Neurology, 70(14), 1208-1214. https://doi.org/10.1212/01.wnl.0000307750.41970.d9.

12. Poels, M. M., Ikram, M. A., van der Lugt, A., Hofman, A., Krestin, G. P., Breteler, M. M., \& Vernooij, M. W. (2011). Incidence of cerebral microbleeds in the general population: the Rotterdam Scan Study. Stroke, 42(3), 656-661. https://doi.org/10.1161/STROKEAHA.110.607184.

13. van Elderen, S. G., Brandts, A., van der Grond, J., Westenberg, J. J., Kroft, L. J., van Buchem, M. A., Smit, J. W., \& de Roos, A. (2011). Cerebral perfusion and aortic stiffness are independent predictors of white matter brain atrophy in type 1 diabetic patients assessed with magnetic resonance imaging. Diabetes care, 34(2), 459-463. https://doi.org/10.2337/dc10-1446.

14. Lee, S. H., Kwon, S. J., Kim, K. S., Yoon, B. W., \& Roh, J. K. (2004). Cerebral microbleeds in patients with hypertensive stroke. Topographical distribution in the supratentorial area. Journal of neurology, 251(10), 1183-1189. https://doi.org/10.1007/s00415-004-0500-6.

15. Lee, S. H., Bae, H. J., Ko, S. B., Kim, H., Yoon, B. W., \& Roh, J. K. (2004). Comparative analysis of the spatial distribution and severity of cerebral microbleeds and old lacunes. Journal of neurology, neurosurgery, and psychiatry, 75(3), 423-427. https://doi.org/10.1136/jnnp.2003.015990.

16. Lee, S. H., Kwon, S. J., Kim, K. S., Yoon, B. W., \& Roh, J. K. (2004). Topographical distribution of pontocerebellar microbleeds. AJNR. American journal of neuroradiology, 25(8), 1337-1341.

17. Kim, B. J., \& Lee, S. H. (2013). Cerebral microbleeds: their associated factors, radiologic findings, and clinical implications. Journal of stroke, 15(3), 153-163. https://doi.org/10.5853/jos.2013.15.3.153.

18. Lee, S. H., Ryu, W. S., \& Roh, J. K. (2009). Cerebral microbleeds are a risk factor for warfarin related intracerebral hemorrhage. Neurology, 72, 171-176.

https://doi.org/10.1212/01.wnl.0000339060.11702.dd.

19. Zhang, H., Deng, J., Sun, N., Zou, L., Han, J., Wei, C., \& He, Y. (2020). Effect of coagulation function on cerebral microbleeds in intracerebral hemorrhage. Brain and behavior, 10(6), e01634. https://doi.org/10.1002/brb3.1634.

20. Liu, J., Wang, D., Xiong, Y., Liu, B., Lin, J., Zhang, S., ... Liu, M. (2017). Association between coagulation function and cerebral microbleeds in ischemic stroke patients with atrial fibrillation and/or rheumatic heart disease. Aging and Disease, 8, 131-135.

https://doi.org/10.14336/AD.2016.0715.

21. Wong, K. S., Chan, Y. L., Liu, J. Y., Gao, S., \& Lam, W. W. (2003). Asymptomatic microbleeds as a risk factor for aspirin-associated intracerebral hemorrhages. Neurology, 60(3), 511-513. https://doi.org/10.1212/01.wnl.0000046583.40125.20.

22. Vernooij, M. W., Haag, M. D., van der Lugt, A., Hofman, A., Krestin, G. P., Stricker, B. H., \& Breteler, M. M. (2009). Use of antithrombotic drugs and the presence of cerebral microbleeds: the Rotterdam Scan Study. Archives of neurology, 66(6), 714-720. https://doi.org/10.1001/archneurol.2009.42.

\section{Tables}


Table 1

Comparison of the baseline characteristics between CMBs group and non-CMBs group

\begin{tabular}{|c|c|c|c|c|}
\hline & CMBs group & Non-CMBs group & t or $\chi^{2}$ value & $P$ value \\
\hline $\begin{array}{l}\text { Number of cases } \\
\text { (n) }\end{array}$ & 121 & 72 & & \\
\hline Age (years) & $67.29 \pm 11.20$ & $61.70 \pm 13.80$ & $2.225^{\mathrm{a}}$ & 0.05 \\
\hline Male, n (\%) & $77(65.8)$ & $47(65.3)$ & $0.006^{b}$ & 0.940 \\
\hline $\begin{array}{l}\text { Hypertension, } \\
\text { n (\%) }\end{array}$ & $89(76.7)$ & $56(82.4)$ & $0.813^{b}$ & 0.365 \\
\hline Diabetes, n (\%) & 39(33.3) & $15(20.2)$ & $1.486^{\mathrm{b}}$ & 0.223 \\
\hline $\begin{array}{l}\text { Systolic BP } \\
(\mathrm{mmHg})\end{array}$ & $152.18 \pm 21.58$ & $157.46 \pm 17.01$ & $-0.726^{a}$ & 0.728 \\
\hline $\begin{array}{l}\text { Diastolic BP } \\
(\mathrm{mmHg})\end{array}$ & $91.18 \pm 11.62$ & $91.62 \pm 13.15$ & $-0.095^{a}$ & 0.925 \\
\hline AT & $38(28.0)$ & $12(13.7)$ & $6.062^{b}$ & 0.014 \\
\hline Current smoking,n(\%) & $27(23.5)$ & $20(27.3)$ & $0.082^{b}$ & 0.775 \\
\hline $\mathrm{BMI},\left(\mathrm{kg} / \mathrm{m}^{2}\right)$ & $29.06 \pm 2.98$ & $28.31 \pm 3.50$ & $0.660^{\mathrm{a}}$ & 0.410 \\
\hline IS & $73(60.8)$ & 27(38.2) & $7.783^{\mathrm{b}}$ & 0.009 \\
\hline BA & $31(25.6)$ & $8(10.9)$ & $4.928^{b}$ & 0.043 \\
\hline \multicolumn{5}{|c|}{$\begin{array}{l}\text { Abbreviations: AT, history of antithrombotic treatment;IS,ischemical stroke;BP, blood pressure; CMBs, } \\
\text { cerebral microbleeds;BA,brain atrophy. }\end{array}$} \\
\hline \multicolumn{5}{|l|}{${ }^{\mathrm{a} C o n d u c t} \mathrm{t}$ test. } \\
\hline${ }^{\mathrm{b}}$ Conduct chi-square te & & & & \\
\hline
\end{tabular}


Table 2

Coagulation function and other blood indicators of the CMBs group and the non-CMBs group

\begin{tabular}{|lllll|}
\hline Risk factor & CMBs group & Non-CMBs group & t or z value & P value \\
\hline PLT $\left(10^{9} / \mathrm{L}\right)$ & $218.21 \pm 73.34$ & $216.04 \pm 83.31$ & $0.140^{\mathrm{a}}$ & 0.399 \\
\hline PT $(\mathrm{s})$ & $11.60(11.00,12.30)$ & $11.30(10.83,12.10)$ & $-1.747^{\mathrm{b}}$ & 0.081 \\
\hline INR & $1.06(0.96,1.12)$ & $0.97(0.93,1.03)$ & $-2.109^{\mathrm{b}}$ & 0.035 \\
\hline APTT (s) & $27.10(25.70,29.30)$ & $27.70(26.20,30.08)$ & $-1.731^{\mathrm{b}}$ & 0.324 \\
\hline TT (s) & $17.60(17.00,18.85)$ & $18.15(16.90,19.55)$ & $-0.570^{\mathrm{b}}$ & 0.569 \\
\hline FBI (g/L) & $3.21(2.63,3.75)$ & $2.89(2.48,3.58)$ & $-1.455^{\mathrm{b}}$ & 0.146 \\
\hline CR ( $\mu$ mol/L) & $91.27 \pm 26.69$ & $90.91 \pm 61.52$ & $0.021^{\mathrm{a}}$ & 0.255 \\
\hline BUN (mmol/L) & $6.64 \pm 2.35$ & $6.61 \pm 4.85$ & $0.018^{\mathrm{a}}$ & 0.138 \\
\hline ALT (U/L) & $20.85 \pm 11.36$ & $27.78 \pm 23.29$ & $-1.011^{\mathrm{a}}$ & 0.114 \\
\hline AST (U/L) & $22.54 \pm 5.10$ & $26.95 \pm 9.42$ & $-1.690^{\mathrm{a}}$ & 0.242 \\
\hline GLU (mmol/L) & $5.99 \pm 1.81$ & $7.01 \pm 3.96$ & $-0.919^{\mathrm{a}}$ & 0.284 \\
\hline GHB (\%) & $6.09 \pm 1.11$ & $6.46 \pm 1.81$ & $-0.642^{\mathrm{a}}$ & 0.356 \\
\hline UA ( $\mu$ mol/L) & $339.15 \pm 93.51$ & $287.16 \pm 142.76$ & $1.123^{\mathrm{a}}$ & 0.350 \\
\hline HCY & $17.65 \pm 8.39$ & $13.12 \pm 3.02$ & $1.467^{\mathrm{a}}$ & 0.051 \\
\hline $\begin{array}{l}\text { Abbreviations: PLT,platelet;PT, prothrombin time; APTT, activated partial thromboplastin time; INR, } \\
\text { international normalized ratio; TT:thrombin time AST,aspartate aminotransferase; ALT, alanine } \\
\text { aminotransferase;BUN, urea nitrogen; CR, creatinine; FBI, fibrinogen; GHB, glycation hemoglobin; GLU, } \\
\text { fasting blood glucose; UA, blood uric acid;HCY,homocysteine. }\end{array}$ & & & \\
\hline aconduct t test. & & & & \\
\hline bConduct Mann-Whitney U-test & & & \\
\hline
\end{tabular}


Table 3

Multivariate logistic regression analysis to demonstrate independent risk factors associated with CMBs

\begin{tabular}{|lllllll|}
\hline & B & SE & Wald & P value & OR & $95 \% \mathrm{Cl}$ \\
\hline IS & 0.701 & 0.349 & 4.053 & 0.044 & 2.016 & $1.090-3.991$ \\
\hline AT & 0.776 & 0.476 & 2.757 & 0.097 & 2.173 & $0.869-5.433$ \\
\hline BA & 0.867 & 0.493 & 3.088 & 0.079 & 2.380 & $0.905-6.260$ \\
\hline age & -0.34 & 0.016 & 4.402 & 0.036 & 0.967 & $0.936-0.998$ \\
\hline INR & -0.998 & 1.016 & 0.965 & 0.326 & 0.369 & $0.050-2.700$ \\
\hline
\end{tabular}

Figures

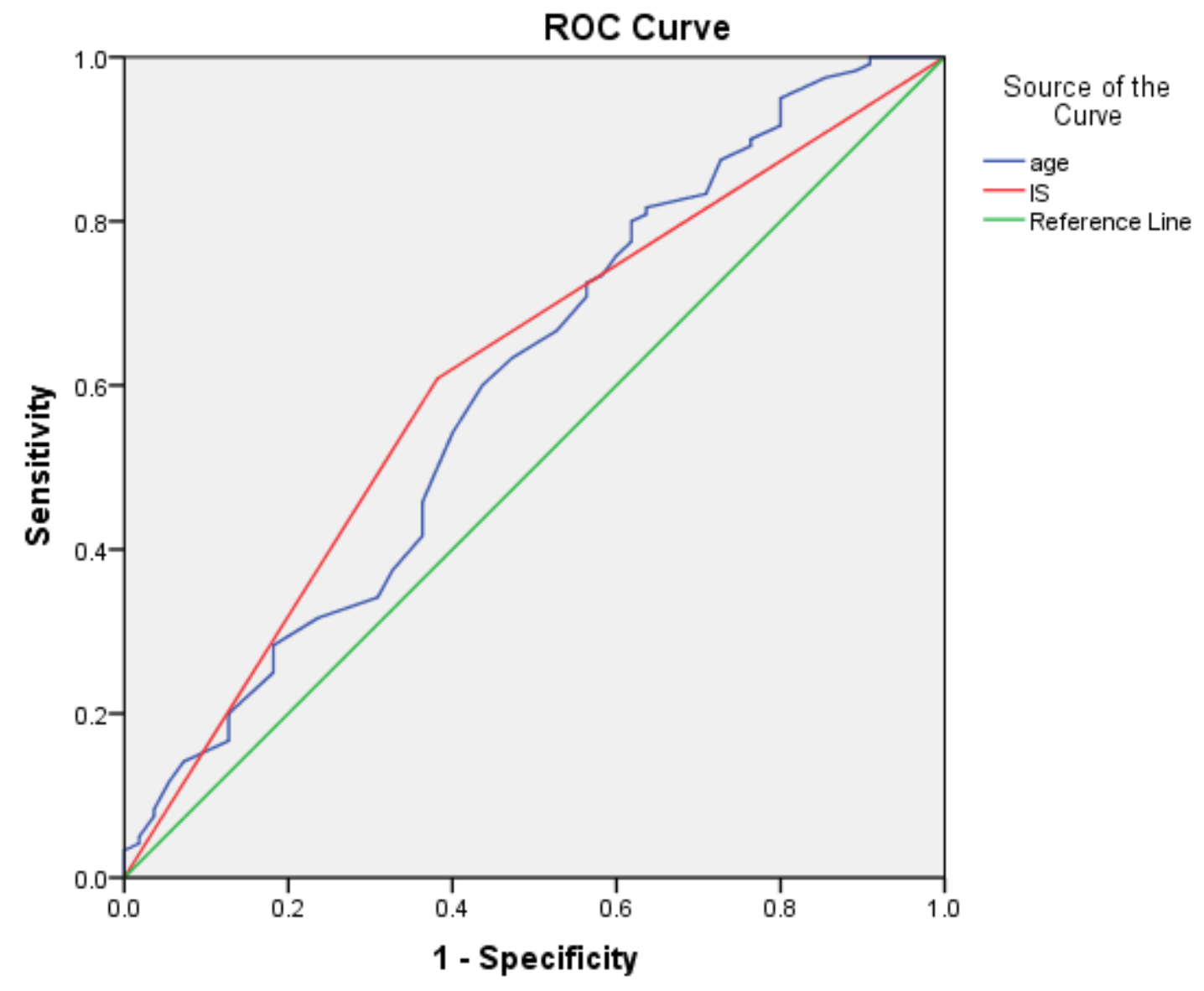

Diagonal segments are produced by ties.

Figure 1 
The ROC curve analysis of age and ischemic stroke(IS) for predicting the occurrence of CMBs in patients with $\mathrm{ICH}$. 